\title{
Window into the Complexities of Chromosome Interactomes
}

\author{
A. Göndör, ${ }^{1}$ A. Fernandez Woodbridge,,${ }^{1,2}$ C. Shi, ${ }^{1}$ E. Aurell,${ }^{2}$ M. Imreh, ${ }^{1}$ And R. Ohlsson ${ }^{1}$ \\ ${ }^{1}$ Department of Microbiology, Tumor and Cell Biology, Karolinska Institute, SE-171 77 Stockholm, Sweden; \\ ${ }^{2}$ Department of Computational Biology, The Royal Institute of Technology, SE-100 44 Stockholm, Sweden \\ Correspondence: Rolf.Ohlsson@ki.se
}

\begin{abstract}
DNA is folded into increasingly complex yet highly mobile structures to organize the chromosomes. In the interphase nucleus, chromosomes or part of the chromosomes encounter one another preferentially at the boundaries between chromosomal territories. Although this situation implies that the preferred chromosomal neighborhood is a key determinant of interactions between chromosomes, what this means in functional terms is currently not well understood. Using the $H 19$ imprinting control region as a window, it has been demonstrated that epigenetic information of the primary chromatin fiber has dual functions. Thus, epigenetic marks not only influence the proximity between chromatin fibers but also transfer epigenetic states between chromatin fibers both in cis and in trans. High-throughput sequence and DNA fluorescence it situ hybridization (FISH) analyses reveal that these features require chromatin movements that are restricted in space and time. The mechanisms involved in the establishment of chromosome interactomes may provide insight of fundamental importance into pivotal regulatory processes in the nucleus, such as the coordination of transcriptional programs and replication timing.
\end{abstract}

The nuclear functions, such as transcription, replication, and DNA repair, are performed in subnuclear compartments, such as the nucleolus and replication and repair "factories" (Göndör and Ohlsson 2009a). These and other nuclear hallmarks are likely organized stochastically by self-assembling factors to provide environments and opportunities for encounters between chromatin fibers (Kaiser et al. 2008). This scenario can be illustrated by the formation of the nucleolus by active ribosomal RNA gene clusters as well as by the simultaneous juxtaposition of active transcriptional units in transcription factories and replicons in replication factories (Göndör and Ohlsson 2009a). From a historical perspective, interactions between chromatin fibers have been known for more than 100 years. Indications of the functionality of such interactions were first hinted at by Drosophila geneticists, who could demonstrate that inactivation of genes could spread over huge distances along the chromosome in cis, and variably from cell to cell, to give rise to variegated gene-silencing effects (Duncan 2002). Moreover, it was also early realized that interactions in trans between two alleles could productively influence expression from a small subset of loci. The term "transvection" was initially invented to explain such complementation between alleles and was subsequently extended to formally include also effects of interactions between different loci (Duncan 2002). These results were obtained using primarily genetic approaches, which, unfortunately, did not allow a further elaboration of the molecular features of chromatin fiber interactions.

A major step forward to explore chromatin fiber interactions at the molecular level was initiated with the invention of the chromosome conformation capture (3C) method (Dekker 2006). The $3 \mathrm{C}$ method has proven to be very useful for determining the close physical proximity of sequences (with a resolution of a few kilobase pairs) from remote intra- or interchromosomal locations. Using this approach, a few seminal discoveries were made to finally advance our knowledge from the fundament provided by Drosophila geneticists. The first study to show a functional link for interactions between chromatin fibers exploited $\mathrm{T}_{\mathrm{H}} 2$ cells for $3 \mathrm{C}$ analysis. Using the $\mathrm{T}_{\mathrm{H}} 2$ locus control region (LCR) as bait, it could be demonstrated that the LCR forms a chromatin hub with the IL4,IL5, and IL13 genes to provide a chromatin conformation poised for transcription (Spilianakis et al. 2005). Subsequently, it was demonstrated that SatB1 is a strong candidate for organizing this chromatin hub before overt transcriptional activation during $\mathrm{T}_{\mathrm{H}} 2$ cell differentiation (Cai et al. 2006). Another example using a variant of the $3 \mathrm{C}$ technique is represented by the striking observation that viral exposure triggers the juxtaposition of Alu repeat segments from different chromosomes with the interferon- $\beta$ locus. Because these Alu elements carry nuclear factor- $\mathrm{\kappa B}$, their interaction allows the formation of the enhanceosome and thus transcriptional activation of the interferon- $\beta$ gene (Apostolou and Thanos 2008).

However, the usefulness of the $3 \mathrm{C}$ method is restricted by the fact that it requires a prior perception of the DNA sequences underlying the two potentially interacting chromatin fibers. Although this limitation can be partially overcome by performing numerous $3 \mathrm{C}$ analyses within a gene cluster, for example (Dostie et al. 2006), it does not readily address the genome-wide perspective. This is not a limitation with the circular (Zhao et al. 2006) or "chip" (Simonis et al. 2006) 3C assay, with the acronym of 4C, which uses a known sequence as a bait to explore all unknown sequences in its physical proximity. This method and its derivatives have been proven very useful to identify the organization of chromosome interactomes linked with epigenetic states and/or transcriptional status. Thus, it could be demonstrated that the active and inactive $\beta$-glo- 
bin LCR has preferential interaction with active and inactive chromosome regions, respectively (Simonis et al. 2006). This is, at least partially, in keeping with the observation that several transcriptional units, even from different chromosomes, colocalize in so-called transcription factories during simultaneous transcription (Schoenfelder et al. 2010). The 4C technique has also been used to demonstrate allelic interactions between the $H 19$ imprinting control region and imprinted and nonimprinted domains on other chromosomes (Zhao et al. 2006; Sandhu et al. 2009).

More recently, a 3C-derived technique, termed $\mathrm{Hi}-\mathrm{C}$, claims to provide an all-to-all map of chromatin fiber interactions, although the resolution is almost at a light microscopic level: $\sim 1 \mathrm{Mb}$ and more represents a manyto-many approach (Lieberman-Aiden et al. 2009). Although this and all other similar studies of physical proximities of chromatin fibers have provided a large amount of new and important information of more or less stochastic encounters between chromatin fibers, it is as yet unknown what this means in functional terms. Although there could be a trivial explanation that inter- and intrachromosomal interactions are nonproductive and simply result from random collisions in the nucleus, available data indicate that at least a subset of chromatin fiber encounters is involved in central nuclear processes, such as the transcription and silencing alluded to above. To assess more broadly the relevance of chromatin fiber interactions, there will be a need to take into account the entire repertoire of nuclear processes, including replication and RNA processing and how these interactions function in the context of the nuclear architecture. Although it can be perceived that some regions, such as LCRs and chromatin insulator regions, may primarily have a role in transcriptional coordination, others, such as replication origins, may be coordinating the timing of replication in longrange manners, and so on. Taken together with the frequent observations that chromatin networks are cell-type-specific, the epigenetic dimension must also be incorporated. It is thus probable that the chromatin networks that are uncovered by the " $C$ " techniques to a very large extent depend on the choice of bait(s) as well as cell types and that the current picture is at best fragmentary.

\section{THE BAIT, H19 IMPRINTING CONTROL REGION, AND ITS LONG-RANGE FUNCTION IN CIS}

The choice of the H19 imprinting control region (ICR) as bait for an extensive 4C analysis reflected its performance as a parent of the origin-specific regulator of monoallelic expression of the neighboring Igf2 and $H 19$ genes (Fig. 1). During the last decade, much attention has been invested in the H19 ICR and its ability to function as an allele-specific chromatin insulator to prevent enhancers from communicating with the maternally inherited $I g f 2$ allele (Wallace and Felsenfeld 2007; Hou and Corces 2010; Ohlsson et al. 2010). The conventional definition of chromatin insulators established by Drosophila geneticists states that chromatin insulators must be positioned between enhancers and promoters to establish an inactive state (Hou and Corces 2010). Thus, targeted insertion of the endogenous endoderm-specific enhancer between Igf2 and the H19 ICR completely neutralizes the insulator function during mouse embryogenesis (Webber et al. 1998), which is in keeping with the position-dependent feature of the chromatin insulator function. Moreover, targeted deletion or mutation of key cis regulatory elements for the 11 zinc-finger protein CTCF leads to loss of insulator function (Pant et al. 2003). Because similar robust assessments have not been done with any other mammalian chromatin insulator, the H19 ICR is, in fact, the only known vertebrate endogenous insulator that conforms to the in vivo definition.

Although the mechanism of chromatin insulation is poorly understood, it is likely to involve interaction between chromatin fibers. Using $3 \mathrm{C}$ assays, others and we have documented that DMR1 and MAR3 represent two key regions that specifically interact with the H19 ICR on the maternal chromosome (Fig. 1) (Murrell et al. 2004; Kurukuti et al. 2006). DMR1 is of particular importance because it has been shown to be essential to repress expression of the maternal Igf2 allele. Thus, targeted deletion of this region leads to derepression of maternal $\operatorname{Igf} 2$ despite the insulator domain being intact (Constancia et al. 2000). This DMR1 property has been postulated to involve

A

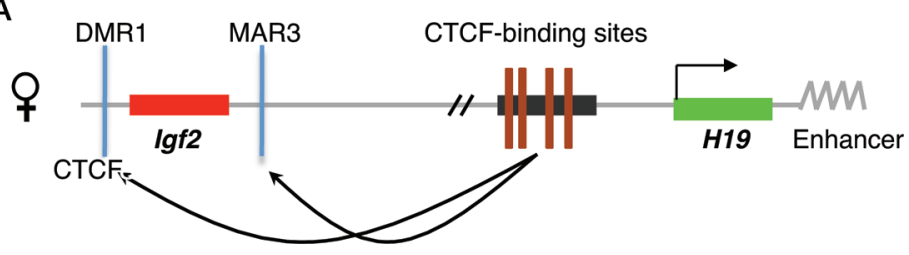

B

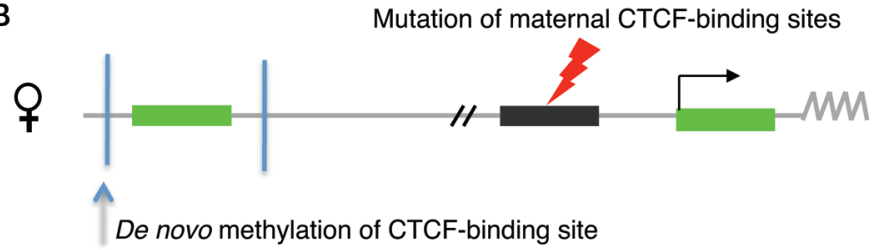

Figure 1. Paradigm of genomic imprinting. $(A)$ Physical proximity between $H 19$ ICR and differentially methylated region (DMR)-1 and matrix-associated region (MAR)-3 on maternal chromosome. (B) Maternal inheritance of mutated H19 ICR allele, deficient in CTCF binding, leads to loss of these interactions and de novo methylation of a CTCF-binding site within the DMR1 (Kurukuti et al. 2006). 
a silencer function that is active only when DMR1 is unmethylated on the maternal allele. Strikingly, mutation of the CTCF-binding sites at the H19 ICR leads to de novo methylation of a CTCF-binding site within DMR1 (Fig. 1), hinting at the possibility that the long-range insulation by CTCF-complexed H19 ICR involves protection against de novo methylation of DMR1 (Kurukuti et al. 2006). This view was supported by the demonstrations that the cohesin complex extensively maps to CTCF-binding sites genomewide and that its down-regulation led to disruption of the DMR1-H19 ICR interaction (Nativio et al. 2009).

It should be kept in mind that although these later results appear to confirm CTCF as a chromatin insulator-specific protein, other explanations cannot as yet be excluded. Apart from being linked with chromatin insulation, CTCFbinding sites also provide protection against de novo DNA methylation. It cannot be formally ruled out, therefore, that the protective shield provided by CTCF enables trans-acting factors other than CTCF and with potential insulator functions to interact with the unmethylated H19 ICR Moreover, because CTCF has also been associated with preventing replication during the early $\mathrm{S}$ phase, a question arises whether there is a CTCF-dependent division of labor at the maternal $\mathrm{H} 19 \mathrm{ICR}$ allele. This issue is highlighted by our observation that a mutant $H 19$ ICR allele has three out of four CTCF-binding sites mutated and, in contrast to the previously published $142 *$ allele, remains unmethylated in somatic cells when maternally inherited (R. Ohlsson et al., unpubl.). Using this model system, it could thus be shown that a strong reduction of CTCF binding leads to loss of asynchronous replication timing of the $\mathrm{Igf} / \mathrm{H} 19$ domain but not to loss of the insulator function (R. Ohlsson et al., unpubl.).

Strikingly, 4C analysis reveals that neither of the CTCFbinding sites at the maternal H19 ICR allele is in direct contact with the CTCF-binding site at DMR1 (Fig. 2), suggesting that the cohesin complex is not primarily establishing a CTCF-CTCF communication, at least not in as- sociation with the chromatin insulator function. Moreover, complete loss of CTCF binding at the maternal H19 ICR allele leads to a dramatic reorganization of the chromatin structure around the H19 ICR. Thus, rather than promoting local chromatin loops, they are prevented by the CTCFbinding sites, and, by inference, the cohesin complexes prevent chromatin folding (Fig. 2). It is of note that the chromatin fiber interactions involving CTCF-binding sites 1 and 2 versus binding sites 3 and 4 are not comparable. Because neither of the CTCF-binding sites within the H19 ICR makes direct contact with the DMR1-specific CTCFbinding site, there must be additional features within the H19 ICR that directly or indirectly maintain CTCF binding at the maternal DMR1 allele. Potentially relevant to this discussion is our previous demonstration that nucleosomes are positioned within the maternal H19 ICR allele to generate $\sim 10$ linker regions constitutively available for transacting factors (Kanduri et al. 2002). Because four of those constitute CTCF-binding sites, the remaining linker regions could attract other important factors to the maternal allele under the umbrella of CTCF protection. In addition, we have observed evolutionarily conserved binding sites for several transcription factors that map not only at these linker regions but also within the nucleosomal sequences adjacent to the CTCF-binding sites. Because several of these factors interact with the maternal H19 ICR allele, this region might form a molecular platform for a protein-protein interactome that may influence long-range chromatin fiber communications (O. Israelsson et al., unpubl.).

\section{H19 ICR-DEPENDENT LONG-RANGE FUNCTION IN TRANS}

Although the H19 ICR is traditionally perceived as functioning only as a chromatin insulator to establish the repressed status of the maternal Igf2 allele, there are reasons to assume that this is a too simplistic view. Thus, a mutant H19 ICR allele, deficient in CTCF binding, accel-
A

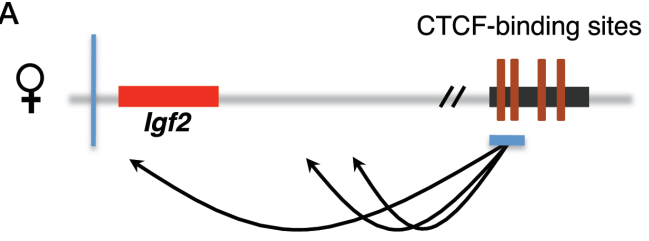

B

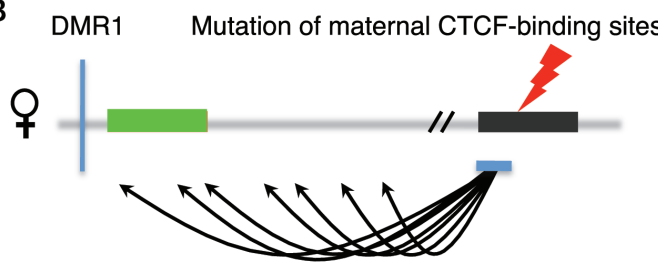

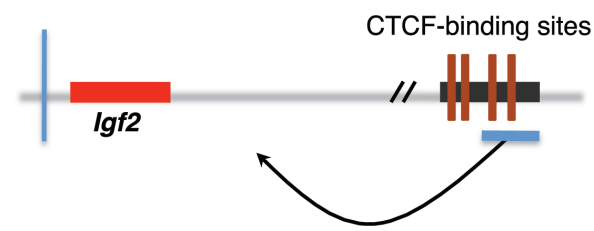

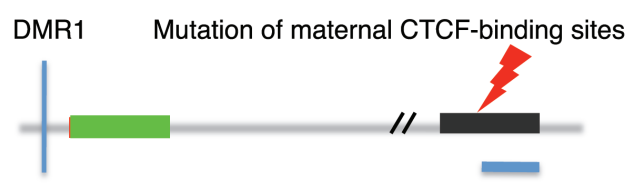

Figure 2. Four-color (4C) analysis of local chromatin loops within Igf2/H19 domain using H19 ICR as bait. Chromatin loops emanating from different parts of $H 19$ ICR are depicted with a maternally inherited wild-type $(A)$ or mutant $(B)$ allele. $(A, B$, Left) Loops emanating from CTCF-binding sites nr 1 and 2; (right) much-less-frequent chromatin fiber interactions from CTCF-binding sites nr 3 and 4. Data were obtained using $4 \mathrm{C}$ probes derived from pools of neonatal liver cells ( 20 million cells in total) hybridized to Nimblegen microarrays with 100 -bp resolution. 
erates the formation of progenitor cells in colon mucosa and predispose to colon cancer only when it is maternally inherited (Sakatani et al. 2005). A similar observation has not been reported with any of the available mouse strains transgenic with $I g f 2$. Moreover, loss of $I g f 2$ imprinting induced by the deletion of CTCF-binding sites on the maternal H19 ICR allele leads to a sensitization to IGF-II signaling (Kaneda et al. 2007). This observation runs counter to the expectations that increased production of IGF-II via biallelic Igf2 expression would eventually attenuate its signaling pathway. It thus appeared clear that this region has a pleiotropic function that goes far beyond merely repressing maternal-specific $I g f 2$ expression.

To explore this contention, we developed the circular chromosome conformation capture (4C) technique and applied it to the H19 ICR (Zhao et al. 2006). In the initial $4 \mathrm{C}$ study, conventional sequencing was performed on cloned 4C samples generated from neonatal liver samples. The identified 4C "interactors," i.e., interacting sequences, were subsequently verified by allele-specific 3C analysis. Although this approach revealed that most of the interactions depended on the CTCF-binding sites of only the maternal H19 ICR allele, both alleles of the bait interacted with the same partner sequence in several instances (Zhao et al. 2006). This interesting result implied that both parental alleles of the H19 ICR were able to communicate with one or both alleles of a region positioned on another chromosome. This feature might, in part, reflect an ability of both parental alleles of the H19 ICR to dynamically explore their microenvironment inside and outside of its chromosomal territory. Thus, although most of the chromatin loops occurred within the chromosomal territory, which is the space the chromosome occupies in interphase nuclei, interchromosomal interactions occurred primarily when the H19 ICR alleles were positioned at the edges of their chromosomal territories (Sandhu et al. 2009). These encounters appeared to be under developmental control, because the patterns of intra- and interchromosomal interactions were almost completely exchanged during the differentiation of embryonic stem (ES) cells into embryoid bodies (Sandhu et al. 2009). Thus, epigenetic states are directly or indirectly implicated in the regulation of opportunities for chromosome cross talk.

The use of genome-wide microarrays with a 100-bp resolution to screen for $4 \mathrm{C}$ interactors revealed that a large portion of chromosome 7, as viewed from the H19 ICR perspective, is contracted in ES cells to expand during differentiation into embryoid bodies (Sandhu et al. 2009). This process is paralleled by an increase in the mobility of the H19 ICR region, indicating that its immediate environment undergoes significant changes during the differentiation process. Despite these changes, there is a strong overrepresentation of genes that share the common feature of being genomically imprinted, irrespective of which cell type the $4 \mathrm{C}$ sequences were derived from. Moreover, not only does the majority of all known imprinted genes interact with the H19 ICR, but they also interact with one another to form what we call an "imprintome" that is manifested both in somatic cells and during germline development (Sandhu et al. 2009). These patterns of interactions appear to be very dynamic because the proportion of imprinted loci in close physical proximity rarely exceeded two at a time in somatic cells. Of note, the frequency of proximity between imprinted domains was threefold to fivefold higher in spermatogonia than in somatic cells represented by neonatal liver cells, suggesting that the imprintome is primarily operating in stem cells of the male germline (Sandhu et al. 2009).

\section{TRANSFER OF EPIGENETIC INFORMATION IN TRANS}

The strong overrepresentation of imprinted domains in the $4 \mathrm{C}$ library is likely to directly or indirectly reflect parent-of-origin-specific epigenetic marks at or close to the site of interacting sequences within imprinted domains. Indeed, in a few instances, such as the Impact and Kcnq1 loci, the interacting sequence mapped within regions exhibiting parental-specific DNA methylation patterns (Sandhu et al. 2009). Moreover, it is clear that the observed interaction network depended on CTCF-binding sites within the maternally inherited H19 ICR allele in spermatogonia (Fig. 3) (Sandhu et al. 2009). Intriguingly, the maternal transmission of an H19 ICR allele unable to interact with CTCF disrupted the interactions not only between the H19 ICR and imprinted domains but also between the imprinted domains themselves in spermatogonia (Fig. 3) (Sandhu et al. 2009).

What does this mean in functional terms? Although we do not yet know the answer to this question, it is intriguing that the maternal H19 ICR is causally linked with the protection against de novo methylation of another CTCFbinding site that is positioned more than $100 \mathrm{~kb}$ away (Fig. 1) (Kurukuti et al. 2006). However, the maternal H19 ICR is able to confer epigenetic marks much farther away than this. The differential replication timing of the parental alleles appears to be a hallmark of imprinted domains and monoallelically expressed genes (Göndör and Ohlsson 2009b). As could be expected from this link, the interacting imprinted regions identified by the $4 \mathrm{C}$ analysis displayed hallmarks of asynchronous replication timing, likely reflecting that the parental alleles replicate early and late, respectively. Importantly, the asynchronously replication-timing patterns of these interactors were lost when the maternal H19 ICR allele carried mutated CTCF-binding sites. Moreover, the late-to-early switch in replication timing in response to mutation of the CTCF-binding sites within the maternal H19 ICR allele in neonatal liver cells (Sandhu et al. 2009) suggests a systemic link between latereplicating alleles that impinges on the late-replicating, maternal H19 ICR allele (Fig. 3). Because replication timing is an epigenetic mark that has been linked to the stable propagation of active and inactive domains (Göndör and Ohlsson 2009b), it can be concluded that the H19 ICR not only has a major role in the formation of the imprintome but also is able to confer or transvect epigenetic states to members of the imprintome.

These observations have many potential ramifications. First, the imprintome, which is conserved between mouse and man (R. Ohlsson et al., unpubl.), could reflect a 

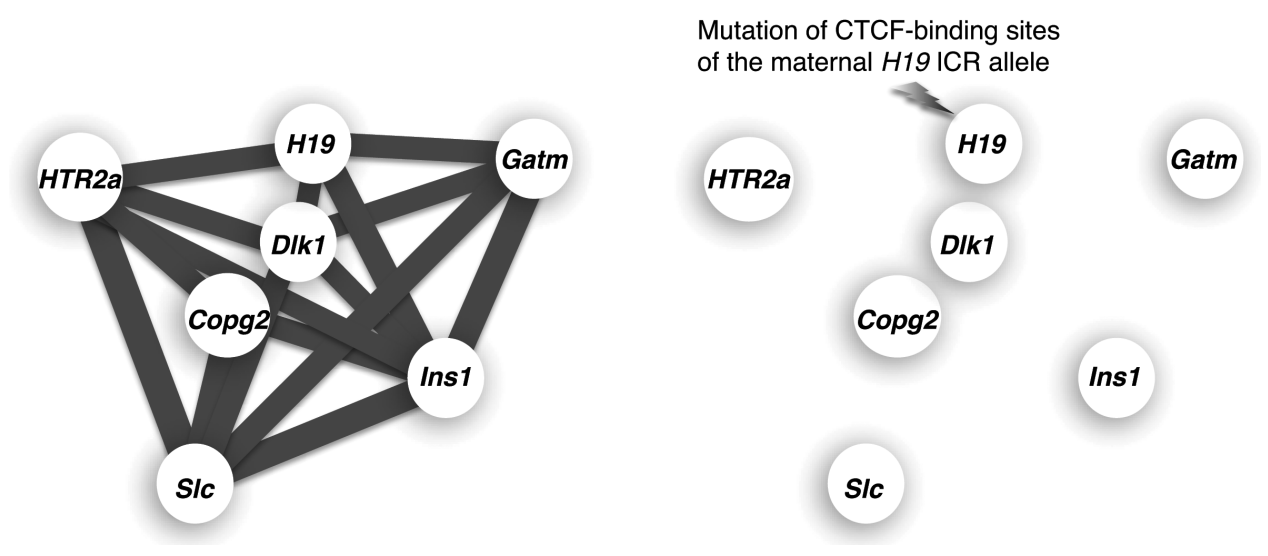

Figure 3. CTCF-dependent interchromosomal network between imprinted domains. Documented pattern of physical interactions among a selection of imprinted domains in spermatogonia and somatic cells. Maternal, but not paternal, inheritance of mutated $H 19$ ICR allele, unable to interact with CTCF, breaks up this network of crosswise interactions and transfers epigenetic states to modify replication-timing patterns of interacting sequences in mouse spermatogonia (Sandhu et al. 2009).

process of a germline-specific recruitment of imprinted domains from a nodal point during early mammalian radiation. Because the H19 ICR interacts with the majority of imprinted domains in the mouse and indirectly controls also interactions between other imprinted domains, it is a candidate for organizing such a node (Sumida and Ohlsson 2010). This perspective is supported by the identification of the H19 ICR as the oldest known imprinting control region. Moreover, most imprinted domains consist of singletons in marsupials, where the imprinted $\mathrm{Ig} 2 \mathrm{f}-\mathrm{H} 19$ cluster stands out. Although speculative, it is thus formally possible that imprinted domains acquired their properties as a result of germline-specific interactions in cis and in trans with the H19 ICR. Under this scenario, the imprintome (Zhao et al. 2006; Sandhu et al. 2009) represents a "living fossil" that may still be operative to reflect an ongoing process (Sumida and Ohlsson 2010).

Second, the parent-of-origin-specific replication-timing feature that is controlled at a distance may explain why imprinted domains are clustered. Thus, an entire domain containing several genes placed in a transcription-permissive (early S phase) environment shows a tendency for being active and attaining active chromatin marks, and vice versa for late-replicating domains (Göndör and Ohlsson 2009b). Of course, this is a simplified view because imprinted clusters can contain active genes on opposite parental chromosomes replicating late and early, respectively. Nonetheless, the CTCF-binding sites on the maternal H19 ICR allele appear to confer a late-replicating feature of not only the Igf2/H19 region (Bergstrom et al. 2007) but also the entire domain of imprinted genes on distal chromosome 7 in the mouse (R. Ohlsson et al., unpubl.). It is possible that the replication-timing character had an important role during the early stages of imprint acquisition, but that additional epigenetic events became manifested to enable escape from the epigenetic confines provided by the replication-timing feature. We thus hypothesize that an ancient H19 ICR of the maternal genome gradually affected epigenetic modifications via long-range interactions to control replication timing in a CTCF-de- pendent manner to facilitate the creation of clusters of imprinted genes in the mammalian genome.

Third, because the network of imprinted domains was disrupted in spermatogonia only when the mutant (CTCFbinding deficient) $H 19$ ICR allele was inherited maternally (Fig. 3), it appears that only maternal-specific marks underlie the ability of imprinted domains to seek out and find one another. This notion is reinforced by the observation that the $H 19$ ICR directly interacts with the Kcnq 1 ICR on the maternal chromosome in the mouse (Sandhu et al. 2009). Similarly, the only known two regions preventing parthenogenesis, H19/Igf2 and Dlk1/Dio3, interact both in the germline and in somatic cells, and the replication-timing pattern of the Dlk1/Dio3 domain is controlled by CTCF-binding sites within the maternal H19 ICR allele (Sandhu et al. 2009).

\section{CHROMOSOME INTERACTOME BEYOND H19 ICR}

Although the chromatin networks that have been described earlier have provided useful information regarding genome architecture, they are limited in that they view one interactor at a time from the bait perspective. This has been partially overcome with the circular 3C method because of its ability frequently to capture more than one interactor at any given time (Fig. 4). By piecing together a host of such interactors, identified by high-throughput sequencing techniques, it is possible to organize a chromosome interactome that represents the sum of all possible interactions also between interactors in a population of cells (Fig. 4). To explore such a chromosome interactome from the window of the H19 ICR and to explore whether or not the chromosome environment around the H19 ICR is evolutionarily stable, we turned our attention from mouse to human embryonic stem cells. Using a BglII fragment within the H19 ICR as bait, a wide range of interactors could be identified by the 454 sequencing technology. The use of the Roche 454 platform rather than Illumina's Solexa platform was motivated by the need to obtain long 
A Chromosome interactome

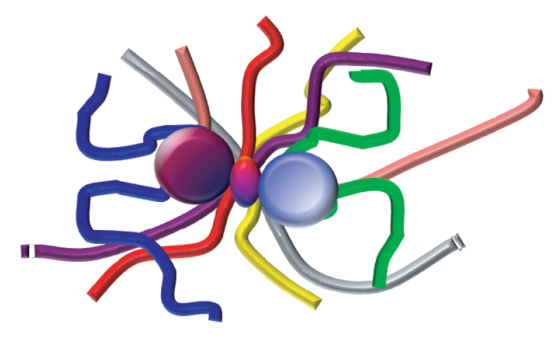

B Chimeric $4 \mathrm{C}$ products

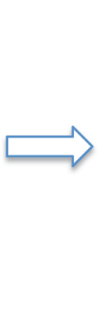

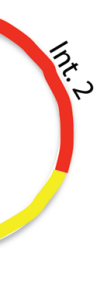

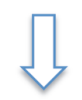

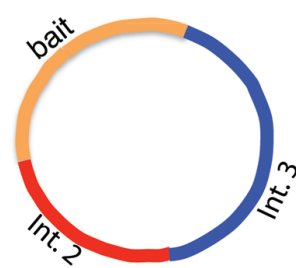

Int. 1
D

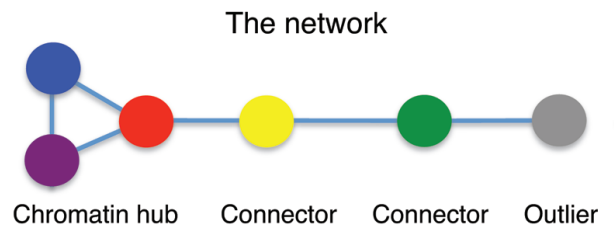

C

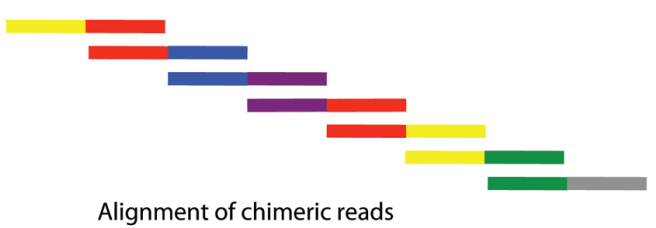

Figure 4. Principle of three-way interactions enables visualization of chromosome interactomes. When two or more members of chromatin hubs $(A)$ can be ligated to the bait $(B)$, high-throughput sequencing $(C)$ can be exploited to organize an interactome $(D)$. Although this interactome will always be viewed from the perspective of the bait, it is very useful to identify interactions between chromatin hubs, their connectors, and outliers.

sequence reads to facilitate the mapping strategy. Indeed, $>90 \%$ of the sequence reads, schematically illustrated in the circus plot of Figure 5A, could be robustly mapped to the human genome.

Exploring the bridges between interacting chromatin fibers containing three or more interactions including the bait - three-way as opposed to two-way - it could be concluded that chromosomal networks can form modular in- teractomes by establishing nodes with different levels of connectivity. Thus, four nodes that interact crosswise with one another and with other nodes with varying degrees of connectivity could be identified as a central hub (Fig. 5B, blue spheres). DNA FISH analysis confirmed that although part of this pattern exists without simultaneously interacting with the H19 ICR, some nodes contribute to the network only in the presence of the H19 ICR (R. Ohls-
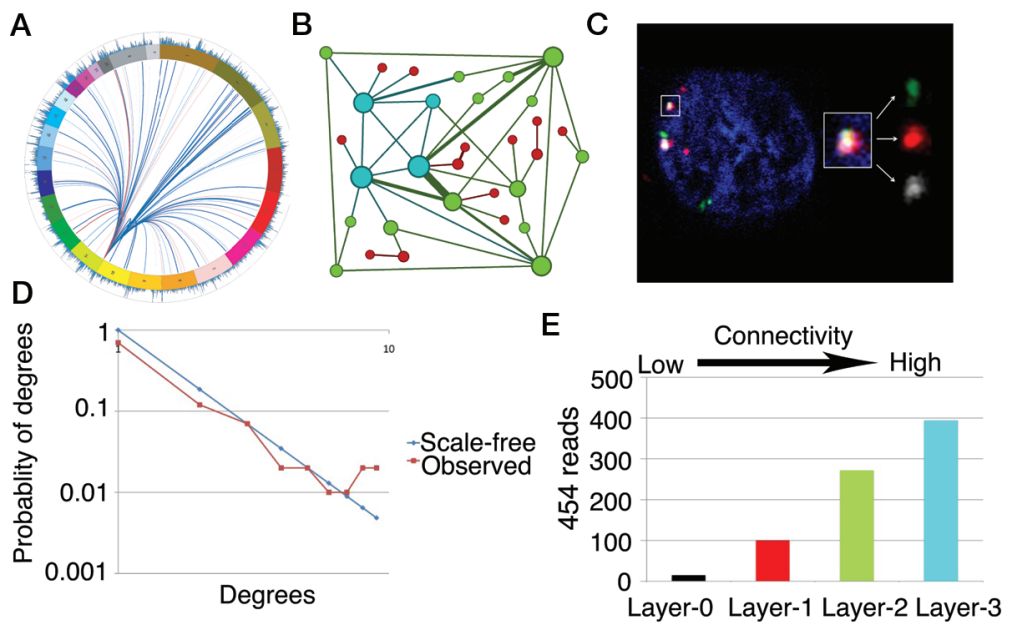

Figure 5. Chromatin hubs interact with one another. $(A)$ Circos plot visualizing genome-wide, two-way interactions in embryoid bodies with H19 ICR as bait. (B) Three-way interactions in human embryoid bodies seen from H19 ICR perspective. Different layers of connectivity are color-coded red, green, and blue with increasing degree of connectivity. Lines represent physical interactions between nodes. Circle size indicates number of connections or degrees that it entertains. Central hub (blue spheres) is maintained by crosswise interactions among the four nodes, which are all LINE-1 elements. (C) DNA FISH analysis showing biallelic clustering of central nodes. For convenience, shown are two collected nodes collected (red and green) and central node with highest connectivity (white). $($ Right $)$ The different regions are visualized in enlarged images. Although the left image was flattened for practical reasons, right images are in the same focal plane. $(D)$ Power-law analysis of three-way network $(B)$, indicating its scale-free character. $(E)$ Comparison of number of total reads for each interacting sequence in indicated categories ( $y$ axis) and color-coded interactome $(B)$ hierarchy $(x$ axis) in human embryoid bodies. 
son et al., unpubl.). Moreover, these chromatin hubs reflect a hierarchal structure as determined by the K-shell/core method with the central hub interacting with one another at the nuclear membrane of human embryoid cells (Fig. 5C). Importantly, power-law analysis indicates that both the two-way and three-way networks are scale-free (Fig. 5D) (data not shown). This hierarchal perspective of chromatin networks is reinforced by our unpublished observation that the total number of 454 reads and the connectivity of the network directly correlate (Fig. 5E). The scale-free character is intrinsic to most regulated networks (Barabasi and Albert 1999; Maslov and Sneppen 2002), suggesting that this network was shaped by evolution rather than by aleatory forces. However, this evolution must take into consideration the fact that chromosomal neighborhoods are likely to change because of changes in chromosome organization. Indeed, comparing the interactome from both human and mouse ES cells and embryoid body cells, it is clear that its composition is not strongly conserved during evolution beyond the imprintome.

\section{CONCLUSIONS}

We conclude that the $H 19$ imprinting control region harbors unique properties and is able to transvect epigenetic states via chromatin networks during female germline development and consequently must be reprogrammed during male germline development. This, in turn, renders it a leading candidate for initiating and implementing the evolution of imprinted states in gene clusters within and between chromosomes. Given that this network includes many nonimprinted loci in ES cells and somatic cells, it is possible that the H19 ICR regulates the expressivity of the genome beyond the imprinted domains. Under this scenario, the mammalian epigenome would display a previously unrecognized degree of functional plasticity. Future data will provide a higher coverage to help us explore the network structure and the relationship between the connectivity and read count of the network members. The prediction is that although the chromosome interactome is physically robust, it is amenable to developmental regulation by targeting members of the node hub. It can thus be hypothesized that these features are dynamic and depend on cell-type-specific epigenetic states of the underlying chromatin targeted by chromatin-remodeling complexes, for example. This contention is supported by our observation that the nuclearmembrane-specific nodes (see above) are cell-type-specific and exclusively map within LINE-1 elements, which are known to reflect epigenetic reprogramming events. Studies of the chromosome interactomes from the epigenetic perspective might thus reveal major new perspectives of how the formation of active, silenced, or poised states can be spread throughout the genome to compound our current understanding of the regulation of gene expression during both normal development and diseases.

\section{ACKNOWLEDGMENTS}

This work was supported by the Swedish Science Research Council, the Swedish Cancer Research Foundation, the Swedish Pediatric Cancer Foundation, the Lundberg Foundation, High-Throughput Epigenetic Regulatory Organization in Chromatin (HEROIC), as well as Chromatin Immunolinked Ligation (ChILL) (EU integrated projects).

\section{REFERENCES}

Apostolou E, Thanos D. 2008. Virus infection induces NF- $\kappa B-d e-$ pendent interchromosomal associations mediating monoallelic IFN- $\beta$ gene expression. Cell 134: 85-96.

Barabasi AL, Albert R. 1999. Emergence of scaling in random networks. Science 286: 509-512.

Bergstrom R, Whitehead J, Kurukuti S, Ohlsson R. 2007. CTCF regulates asynchronous replication of the imprinted $H 19 / \operatorname{Ig} 2$ domain. Cell Cycle 6: 450-454.

Cai S, Lee CC, Kohwi-Shigematsu T. 2006. SATB1 packages densely looped, transcriptionally active chromatin for coordinated expression of cytokine genes. Nat Genet 38: 1278-1288.

Constancia M, Dean W, Lopes S, Moore T, Kelsey G, Reik W. 2000. Deletion of a silencer element in Igf2 results in loss of imprinting independent of H19. Nat Genet 26: 203-206.

Dekker J. 2006. The three 'C's of chromosome conformation capture: Controls, controls, controls. Nat Methods 3: 17-21.

Dostie J, Richmond TA, Arnaout RA, Selzer RR, Lee WL, Honan TA, Rubio ED, Krumm A, Lamb J, Nusbaum C, et al. 2006. Chromosome Conformation Capture Carbon Copy (5C): A massively parallel solution for mapping interactions between genomic elements. Genome Res 16: 1299-1309.

Duncan IW. 2002. Transvection effects in Drosophila. Annu Rev Genet 36: 521-556.

Göndör A, Ohlsson R. 2009a. Chromosome crosstalk in three dimensions. Nature 461: 212-217.

Göndör A, Ohlsson R. 2009b. Replication timing and epigenetic reprogramming of gene expression: A two-way relationship? Nat Rev Genet 10: 269-276.

Hou C, Corces VG. 2010. Insulators and imprinting from flies to mammals. BMC Biol 8: 104. doi: 10.1186/1741-7007-8-104.

Kaiser TE, Intine RV, Dundr M. 2008. De novo formation of a subnuclear body. Science 322: 1713-1717.

Kanduri M, Kanduri C, Mariano P, Vostrov AA, Quitschke W, Lobanenkov V, Ohlsson R. 2002. Multiple nucleosome positioning sites regulate the CTCF-mediated insulator function of the H19 imprinting control region. Mol Cell Biol 22: 3339-3344.

Kaneda A, Wang CJ, Cheong R, Timp W, Onyango P, Wen B, Iacobuzio-Donahue CA, Ohlsson R, Andraos R, Pearson MA, et al. 2007. Enhanced sensitivity to IGF-II signaling links loss of imprinting of $I G F 2$ to increased cell proliferation and tumor risk. Proc Natl Acad Sci 104: 20926-20931.

Kurukuti S, Tiwari VK, Tavoosidana G, Pugacheva E, Murrell A, Zhao Z, Lobanenkov V, Reik W, Ohlsson R. 2006. CTCF binding at the $H 19$ imprinting control region mediates maternally inherited higher-order chromatin conformation to restrict enhancer access to Igf2. Proc Natl Acad Sci 103: 10684-10689.

Lieberman-Aiden E, van Berkum NL, Williams L, Imakaev M, Ragoczy T, Telling A, Amit I, Lajoie BR, Sabo PJ, Dorschner MO, et al. 2009. Comprehensive mapping of long-range interactions reveals folding principles of the human genome. Science 326: 289-293.

Maslov S, Sneppen K. 2002. Specificity and stability in topology of protein networks. Science 296: 910-913.

Murrell A, Heeson S, Reik W. 2004. Interaction between differentially methylated regions partitions the imprinted genes $\operatorname{Ig} f 2$ and H19 into parent-specific chromatin loops. Nat Genet 36: 889893.

Nativio R, Wendt KS, Ito Y, Huddleston JE, Uribe-Lewis S, Woodfine K, Krueger C, Reik W, Peters JM, Murrell A. 2009. Cohesin is required for higher-order chromatin conformation at the imprinted IGF2-H19 locus. PLoS Genet 5: e1000739. doi: 10.1371/journal.pgen.1000739.

Ohlsson R, Lobanenkov V, Klenova E. 2010. Does CTCF mediate between nuclear organization and gene expression? Bioessays 32: $37-50$. 
Pant V, Mariano P, Kanduri C, Mattsson A, Lobanenkov V, Heuchel R, Ohlsson R. 2003. The nucleotides responsible for the direct physical contact between the chromatin insulator protein CTCF and the H19 imprinting control region manifest parent of origin-specific long-distance insulation and methylation-free domains. Genes Dev 17: 586-590.

Sakatani T, Kaneda A, Iacobuzio-Donahue CA, Carter MG, de Boom Witzel S, Okano H, Ko MS, Ohlsson R, Longo DL, Feinberg AP. 2005. Loss of imprinting of Igf2 alters intestinal maturation and tumorigenesis in mice. Science 307: 1976-1978.

Sandhu KS, Shi C, Sjolinder M, Zhao Z, Göndör A, Liu L, Tiwari VK, Guibert S, Emilsson L, Imreh MP, Ohlsson R. 2009. Nonallelic transvection of multiple imprinted loci is organized by the H19 imprinting control region during germline development. Genes Dev 23: 2598-2603.

Schoenfelder S, Sexton T, Chakalova L, Cope NF, Horton A, Andrews S, Kurukuti S, Mitchell JA, Umlauf D, Dimitrova DS, et al. 2010. Preferential associations between co-regulated genes reveal a transcriptional interactome in erythroid cells. Nat Genet 42: 53-61.
Simonis M, Klous P, Splinter E, Moshkin Y, Willemsen R, de Wit E, van Steensel B, de Laat W. 2006. Nuclear organization of active and inactive chromatin domains uncovered by chromosome conformation capture-on-chip (4C). Nat Genet 38: 1348-1354.

Spilianakis CG, Lalioti MD, Town T, Lee GR, Flavell RA. 2005. Interchromosomal associations between alternatively expressed loci. Nature 435: 637-645.

Sumida N, Ohlsson R. 2010. Chromosomal networks as mediators of epigenetic states: The maternal genome connection. Epigenetics 5: 297-300.

Wallace JA, Felsenfeld G. 2007. We gather together: Insulators and genome organization. Curr Opin Genet Dev 17: 400-407.

Webber AL, Ingram RS, Levorse JM, Tilghman SM. 1998. Location of enhancers is essential for the imprinting of $H 19$ and Igf2 genes. Nature 391: 711-715.

Zhao Z, Tavoosidana G, Sjolinder M, Göndör A, Mariano P, Wang S, Kanduri C, Lezcano M, Sandhu KS, Singh U, et al. 2006. Circular chromosome conformation capture (4C) uncovers extensive networks of epigenetically regulated intra- and interchromosomal interactions. Nat Genet 38: 1341-1347. 


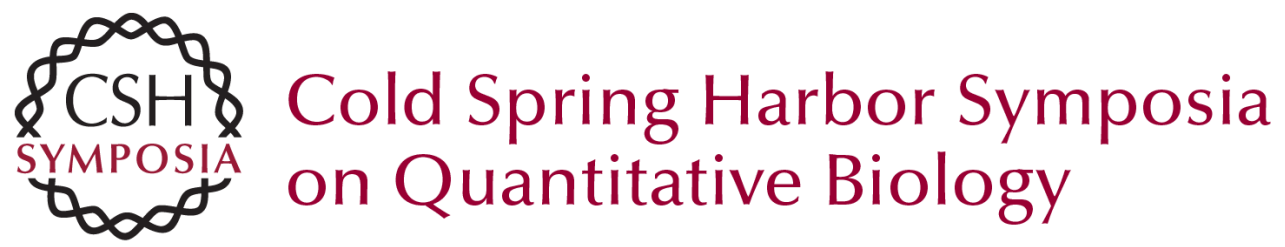

\section{Window into the Complexities of Chromosome Interactomes}

A. Göndör, A. Fernandez Woodbridge, C. Shi, et al.

Cold Spring Harb Symp Quant Biol 2010 75: 493-500 originally published online April 5, 2011

Access the most recent version at doi:10.1101/sqb.2010.75.049

References This article cites 30 articles, 11 of which can be accessed free at:

http://symposium.cshlp.org/content/75/493.full.html\#ref-list-1

License

Email Alerting Receive free email alerts when new articles cite this article - sign up in the box at the Service top right corner of the article or click here.

To subscribe to Cold Spring Harbor Symposia on Quantitative Biology go to:

http://symposium.cshlp.org/subscriptions 